images analysis led to classification of pulmonary segments as "negative" (normal morphology) and "positive" (GGO). Furthermore, the "Warrick score" was used as a staging tool for SSc-ILD. Mean Standardised Uptake Value (mSUV) of segmental parechima was normalised (nmSUV) by comparison with the values of selected control subjets.

Results: No SSc patient was affected by cancer. Three patients had a Warrick Score $>0$, while 4 patients did not had any lung involvement (Warrick Score $=0$ ). The 3 patients with a Warrick Score $>0$ had also skin involvement with a median mRSS 6 (2-7) and pathological lung FDG uptake. In "positive" segments of SSc patients, nmSUV was significantly higher than in the lung segments of the control population (mean estimation 1.53; C.I. 1.42-1.65, p<0.0001). In "negative" segments of SSc patients, with a Warrick score $>0$, the nmSUV was significantly higher than in segments of the control population (mean estimation 1.29; C.I. $1.22-1.37, \mathrm{p}<0.0001)$. Lung segments with GGO showed an nmSUV higher $(21 \%)$ than "negative" segments (C.I. $0.13-0.28, p<0.0001)$ of patients with Warrick score $>0$. "Negative" lung segments of patients with Warrick Score $>0$ showed a $32 \%$ higher 18F-FDG uptake than "negative" lung segments of patients with Warrick Score $=0$. (C.I. 0.17-0.48, p<0.0001). (figure 1)

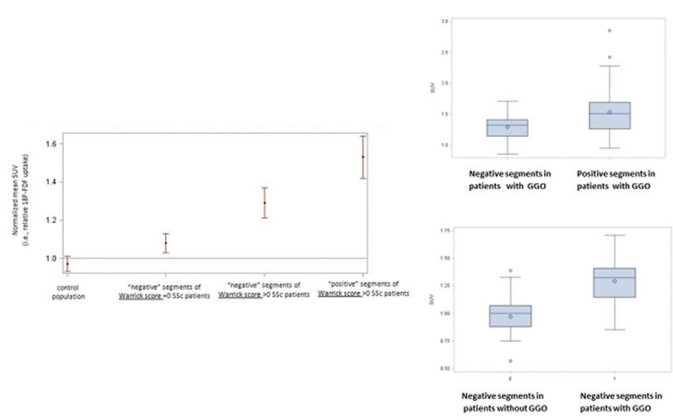

Abstract THU0425 - Figure 1. a) Differences in 18F-FDG uptake between "positive" segments of Warrick score $>0$ SSc patients, "negative" segments of Warrick score $>0$ SSc patients and "negative" segments of Warrick score $=0$ SSc patients vs attended normalised control value (=1); b) Differences in 18F-FDG uptake between "positive" lung segments of SSc patients with Warrick score>0 vs "negative" segments of the same patients; c) Differences in 18F-FDG uptake "negative" lung segments of patients with Warrick Score>0 vs "negative" lung segments of patients with Warrick Score=0

Conclusions: Morphologically "positive" GGO segments show an increased 18F-FDG uptake suggesting the existence of a metabolically active (inflammatory) GGO. However, in patients with GGO, negative lung segments showed a higher nmSUV than negative lung segments in patients without GGO. This may suggest that PET/CT may disclose an underlying inflammatory process, which cannot yet be evidenced by HRCT. Further studies on a larger population are warranted to confirm these data and possibly provide a prognostic significance of $\mathrm{PET} / \mathrm{CT}$ positivity in SSc patients.

Disclosure of Interest: None declared

DOI: 10.1136/annrheumdis-2018-eular.3748

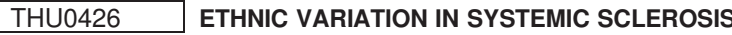 MORBIDITY AND MORTALITY}

H. Al-Sheikh, Z. Ahmad, S.R. Johnson. Toronto Scleroderma Program, Division of Rheumatology, Department of Medicine, Sinai Health Systems, University Health Network, University of Toronto, Toronto, Canada

Background: Systemic sclerosis (SSc) is an uncommon connective tissue disease characterised by pathological skin thickening and can involve multiple internal organs. Ethnic variations in SSc have been reported in clinical manifestations, severity of the disease as well as survival.

Objectives: Our aim was to compare the survival and disease manifestations across ethnicity among SSc patients.

Methods: The Toronto Scleroderma Program is the largest single-centre, multiethnic, longitudinal SSc cohort in Canada. Patients are followed every 6 to 12 months using a standardised protocol. Patients who fulfilled the American College of Rheumatology-European League Against Rheumatism classification criteria for SSc and are 16 years of age or older were included in our retrospective cohort study. The study period was 1970-2017. Ethnicity was self-reported and was categorised as: Caucasian, African-American, Hispanic, Arab, East-Asian, First Nations or Persian. The primary outcome was the time from diagnosis to death from all causes. Secondary outcomes were differences in disease duration, SSc subtype, clinical manifestations, and serology. Survival probabilities and median survival times were determined using Kaplan-Meier survival curves. Cox proportional hazard models were used to estimate adjusted survival.
Results: 1005 subjects were evaluated, the majority of whom were Caucasian $(\mathrm{n}=745(74 \%)$, African-American $\mathrm{n}=58(6 \%))$, South Asian $(\mathrm{n}=69(7 \%))$, and East Asian $(n=80(8 \%))$. Compared to Caucasians, East Asians less frequently had calcinosis $(29 \%$ versus $9 \%, p=0.002)$, and esophageal dysmotility ( $88 \%$ versus $69 \%$, $\mathrm{p}=0.002)$; African-Americans more frequently had interstitial lung disease $(31 \%$ versus $53 \%, p=0.007$ ); and First Nation subjects more frequently had diffuse cutaneous disease ( $35 \%$ versus $56 \%, p=0.02)$ and diabetes $(5 \%$ versus $33 \%$, $\mathrm{p}=0.03$ ). There were no differences across ethnicities in the prevalence of pulmonary hypertension, renal crisis, or digital ulcers.

We found no difference in the short-term survival across ethnicities. However, in the long-term, there was trend for Hispanic subject to have better survival $81.3 \%$ $(95 \% \mathrm{Cl} 63,100)$, while First Nations $(58.3 \%(95 \% \mathrm{Cl} 25,100)$ and South Asian subjects $(52.6 \%(95 \% \mathrm{Cl} 32,87)$ had worst survival at 15 years and 20 years, respectively. East Asians appear to have the longest median survival time 43.3 years.

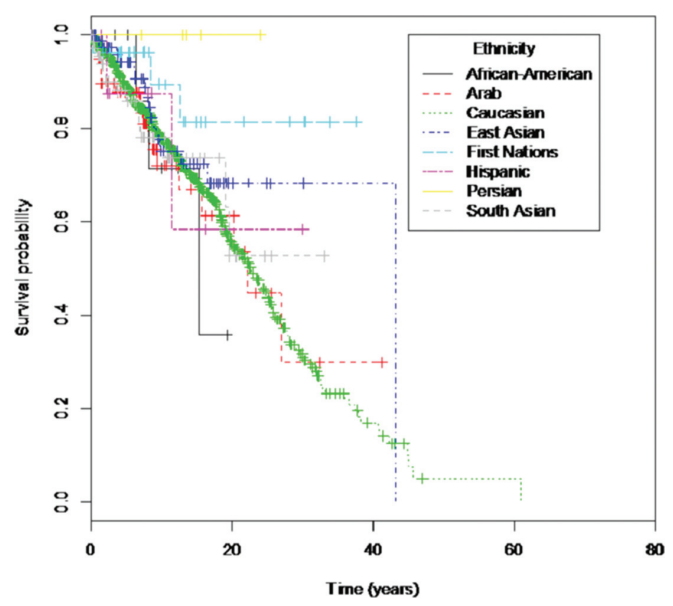

Conclusions: Ethnic variations in disease SSc disease manifestations are observed. However, in the setting of a universal health care system, this does not result in significant differences in survival.

Disclosure of Interest: None declared

DOI: 10.1136/annrheumdis-2018-eular.1489

\section{THU0427 COMPARABLE CARDIOVASCULAR DISEASE AND NEOPLASM RATES BUT HIGHER FREQUENCY OF DEPRESSION IN SYSTEMIC SCLEROSIS VERSUS RHEUMATOID ARTHRITIS: A MULTICENTRE COMPARATIVE STUDY OF COMORBIDITIES}

S. Panopoulos ${ }^{1}$, M.G. Tektonidou ${ }^{1}$, A. Drosos ${ }^{2}$, S.-N. Liossis ${ }^{3}$, T. Dimitroulas ${ }^{4}$ L. Sakkas ${ }^{5}$, D. Boumpas ${ }^{1}$, P. Voulgari ${ }^{2}$, D. Daoussis ${ }^{3}$, K. Thomas ${ }^{1}$, G. Georgiopoulos ${ }^{1}$, G. Vosvotekas ${ }^{4}$, D. Vassilopoulos ${ }^{1}$, P.P. Sfikakis ${ }^{1} .{ }^{1}$ Joint Rheumatology Program, School of Medicine, National and Kapodistrian University of Athens, Athens; ${ }^{2}$ Rheumatology Clinic, Department of Internal Medicine, Medical School, University of loannina, loannina; ${ }^{3}$ Division of Rheumatology, Department of Internal Medicine, Faculty of Medicine, University of Patras, Patras; ${ }^{4} 4$ th Department of Internal Medicine, School of Medicine, Hippokration Hospital, Aristotle University of Thessaloniki, Thessaloniki; ${ }^{5}$ Department of Rheumatology and Clinical Immunology, Faculty of Medicine, School of Health Sciences, University of Thessaly, Larissa, Greece

Background: An increased burden of comorbid conditions negatively impacts patients' outcomes, leads to increased mortality and seems to characterise all chronic systemic connective tissue diseases. Systemic Sclerosis (SSc) is associated with the highest mortality rate comparing to other diseases, whereas data regarding epidemiology and clinical expression of SSc comorbidities is limited. In contrast, comorbidities of rheumatoid arthritis (RA), and especially the increased rate of cardiovascular disease, are better established.

Objectives: To compare the prevalence of common comorbidities in SSc versus RA in a large multicentre case-control study from 5 academic centres in Greece. Methods: Between 2016 and 2017 consecutive SSc patients ( $n=408$, mean age 58.6 years, $88 \%$ women) were matched $1: 1$ for age and gender with 408 RA patients. Evaluated comorbidities were dyslipidemia, diabetes mellitus, arterial hypertension, coronary artery disease, stroke, chronic obstructive pulmonary disease, osteoporosis, neoplasms and depression. Differences were examined by $x^{2}$ test. 